\title{
Epigallocatechin gallate suppresses LPS endocytosis and nitric oxide produc- tion by reducing Rab5-caveolin-1 interaction
}

\author{
Makoto Hagiwara and Kenji Matsushita \\ Department of Oral Disease Research, National Center for Geriatrics and Gerontology, 35 Gengo Morioka-machi, Obu City, Aichi 474- \\ 8511, Japan
}

(Received 26 December 2013; and accepted 31 January 2014)

\begin{abstract}
Epigallocatechin gallate (EGCG) is the major polyphenol in green tea and the main bioactive compound responsible for the health benefits of tea consumption. The molecule exhibits antimicrobial and anti-inflammatory activities. However, little is known about the molecular mechanisms by which EGCG elicits those activities. In this study, we examined the effects of EGCG on lipopolysaccharide (LPS) endocytosis and LPS-induced NO production. We also investigated the mechanism with focus on the effect of EGCG on interaction between small GTPase Rab5 and caveolin-1. EGCG suppressed endocytosis of LPS and LPS-induced nitric oxide production in mouse macrophage-like cells (RAW 264). EGCG suppressed the Rab5-caveolin-1 interaction and reduced Rab5 activity. The results suggest that EGCG reduced LPS-induced innate immune responses through suppression of LPS endocytosis by interfering with Rab5-caveolin-1 interaction and by reducing Rab5 activity.
\end{abstract}

Epigallocatechin gallate (EGCG) is the most abundant catechin in green tea. EGCG has anti-inflammatory, anti-viral, anti-carcinogenic and anti-oxidant activities and may prevent oxidative damage in many organs, including the heart, kidney, lungs and spleen $(14,18)$. However, little is known about the molecular mechanisms by which EGCG exhibits such activities.

Rab5 is a small GTPase localized to early endosomes. It regulates the transport of endocytic vesicles and fusion of early endosomes $(17,24)$. The GTP-bound form of Rab5 is the active form in membrane fusion, and expression of a constitutively active mutant (Rab5Q79L) in cells induces formation of giant endosomes in the cell (22). The GDP-bound form of Rab5 is the inactive form, and expression

Address correspondence to: Kenji Matsushita, Department of Oral Disease Research, National Center for Geriatrics and Gerontology, 35 Gengo, Morioka-machi, Obu city, Aichi 474-8511, Japan

Tel: +81-562-44-5651, Fax: +81-562-46-8479

E-mail: kmatsu30@ncgg.go.jp of a dominant-negative mutant (Rab5S34N) is localized to the cytosol, ubiquitously (15). Activation of Rab5 is facilitated by guanine nucleotide exchange factors (GEFs), whereas inactivation of Rab5 is accelerated by GTPase-activating proteins (GAPs) (21).

Caveolae are specialized lipid rafts that have various signaling functions (3). They are found in a variety of cell types, especially endothelial cells, but none exists as classical invaginated caveolae in neuronal tissues (20). Caveolin-1 is a $25-\mathrm{kDa}$ protein and is a principal component of caveolae membranes. It has been reported that caveolin- 1 is important for caveolae-dependent endocytosis (20). A recent study showed that caveolin-1 interacts with Rab5 and activates Rab5 through direct interaction during caveolae-dependent endocytosis (10).

Lipopolysaccharide (LPS), a membrane protein of gram-negative bacteria, induces secretion of large amounts of several proinflammatory cytokines and proteinases from host cells such as macrophages and evokes inflammation (19). LPS triggers an innate immune response and induces shock in the body (19). 
In this study, we investigated the effect of EGCG on cellular responses to LPS in cultures. EGCG suppressed LPS endocytosis and LPS-induced NO production. We revealed that EGCG decreased Rab5caveolin-1 interaction and reduced Rab5 activity. Our results suggest that EGCG suppresses endocytosis of LPS by decreasing Rab5-caveolin-1 interaction, thereby suppressing LPS-induced NO generation.

\section{MATERIALS AND METHODS}

Specimens. Epigallocatechin gallate (EGCG) was purchased from Sigma-Aldrich (St. Louis, MO).

Antibodies. Antibodies were obtained from the following sources: anti-mouse Rab5 and anti-mouse caveolin-1 (BD Bioscience, Tokyo), anti-rabbit caveolin-1 (Santa Cruz, Dallas, TX), and anti-mouse GFP (anti-mouse YFP) (Novus Biologicals, Littleton, $\mathrm{CO}$ ).

Vector constructs. YFP-Rab5, YFP-Rab5S34N, YFPRab5Q79L and GFP-Rab5 wild in pcDNA3 and CFP-caveolin-1 in pCDNA3.1 constructs were kindly provided by Dr. Y. Yamamoto (Tokyo University of Agriculture, Tokyo) (8-10). The GST-R5BD vector was a kind gift from Dr. G. Li (University of Oklahoma Health Science Center, Oklahoma City, OK) (16).

Stable transformed cells. Human embryonic kidney 293 (HEK293) cells that stably express YFP-Rab5 and CFP-caveolin-1 were obtained using Lipofectamine 2000 (Invitrogen Co., Carlsbad, CA) according to the manufacturer's instructions. HEK293 cells and HEK293 stable cell lines were cultured in Dulbecco's modified Eagle's medium (DMEM) (Wako Pure Chemical Industries, Ltd., Osaka) supplemented with $10 \%$ fetal bovine serum (FBS), $1 \%$ penicillin, and $1 \%$ streptomycin. Cells were observed by confocal fluorescence microscopy (Carl Zeiss Microscopy $\mathrm{GmbH}$, Gottingen, Germany).

Endocytosis assay. To measure the endocytosis of LPS, mouse macrophage-like cells (RAW 264) were pre-incubated with DMEM without a $\mathrm{pH}$ indicator, phenol red, for $1 \mathrm{~h}$ at $37^{\circ} \mathrm{C}$ in 24-well plates and then incubated with $10 \mu \mathrm{g} / \mathrm{mL}$ LPS Alexa Fluor 568 (Invitrogen) diluted with DMEM without phenol red for $1 \mathrm{~h}$ at $37^{\circ} \mathrm{C}$ or $4^{\circ} \mathrm{C}$ to measure the background level of uptake (negative control). After incubation, the cells were collected with ice-cold PBS, washed eight times with ice-cold PBS, lysed with PBS con- taining $1 \%$ Triton $\mathrm{X}-100$, and then centrifuged at $10,000 \times \mathrm{g}$ for $20 \mathrm{~min}$ at $4^{\circ} \mathrm{C}$. Signal intensity of the supernatants was measured using SpectraMax M3 (Molecular Devices).

Measurement of NO. Monolayers of RAW 264 cells were preincubated with serum-free DMEM without phenol red for $1 \mathrm{~h}$ at $37^{\circ} \mathrm{C}$ in 96 -well plates. The cells were subsequently incubated with $100 \mathrm{ng} / \mathrm{mL}$ LPS (Sigma-Aldrich, Buchs, SG) and $100 \mu \mathrm{g} / \mathrm{mL}$ EGCG for $6 \mathrm{~h}$ at $37^{\circ} \mathrm{C}$. The cells were then incubated with $10 \mu \mathrm{M}$ Diaminofluorescein-2 (DAF-2) (Sekisui Medical Co., Ltd., Tokyo), a sensitive fluorescent indicator for the detection of $\mathrm{NO}$, for $1 \mathrm{~h}$ at $37^{\circ} \mathrm{C}$. Fluorescence intensity of the media was measured using SpectraMax M3 (Molecular Devices, Sunnyvale, $\mathrm{CA}$ ).

Measurement of Rab5-caveolin-1 interaction by FRET. HEK293 stable cell lines expressing YFPRab5 and CFP-caveolin-1 were washed four times with ice-cold PBS, collected with ice-cold PBS, homogenized with PBS, and then centrifuged at 10,000 $\times g$ for $20 \mathrm{~min}$ at $4{ }^{\circ} \mathrm{C}$. The supernatant was added to 96-well black plates with or without EGCG. FRET efficiency of the cell lysate was measured using SpectraMax M3 (Molecular Devices).

Immunoprecipitation. HEK 293 cells were treated with EGCG and lysed for $30 \mathrm{~min}$ at $4^{\circ} \mathrm{C}$ with a buffer $(10 \mathrm{mM}$ Tris, $\mathrm{pH} 7.6,150 \mathrm{mM} \mathrm{NaCl}, 5 \mathrm{mM}$ $\mathrm{MgCl}_{2}, 1 \%$ NP-40, $0.5 \mu \mathrm{g} / \mathrm{mL}$ leupeptin, $2 \mu \mathrm{g} / \mathrm{mL}$ aprotinin, and $10 \mu \mathrm{g} / \mathrm{mL}$ PMSF). The clarified lysates were incubated with anti-caveolin-1 antibody for $2 \mathrm{~h}$ at $4^{\circ} \mathrm{C}$. The immune complexes were precipitated with protein A-Sepharose beads (Millipore) for $2 \mathrm{~h}$ at $4^{\circ} \mathrm{C}$ and then washed extensively with lysis buffer. The protein A-Sepharose beads were resuspended in SDS sample buffer and assayed by Western blotting.

GST-R5BD pull-down assay. The GST-R5BD pulldown assay was based on the method described by Liu et al. (16). HEK 293 cells transfected with GFP-Rab5 wild were treated with $100 \mu \mathrm{g} / \mathrm{mL}$ EGCG for $2 \mathrm{~h}$ and then washed twice with PBS and lysed for $5 \mathrm{~min}$ in $1 \mathrm{~mL}$ of a lysis buffer $(25 \mathrm{mM}$ HEPES, $\mathrm{pH} 7.4,100 \mathrm{mM} \mathrm{NaCl}, 5 \mathrm{mM} \mathrm{MgCl}_{2}, 0.1 \% \mathrm{NP}-40$, $2 \%$ glycerol, and protease inhibitor). The cell extracts were clarified by centrifugation at $10,000 \times g$ for $5 \mathrm{~min}$ at $4^{\circ} \mathrm{C}$, and the supernatants were incubated with $20 \mu \mathrm{L}$ of GST-R5BD bound to GlutathioneSepharose $4 \mathrm{~B}$ beads for $20 \mathrm{~min}$ at $4^{\circ} \mathrm{C}$ with rotation. 
The beads were subsequently rinsed with lysis buffer, resuspended in SDS sample buffer, and then analyzed by Western blotting.

\section{RESULTS}

EGCG suppresses LPS endocytosis and LPS-induced nitric oxide production

LPS must be internalized to facilitate endotoxindependent signal activation in cells (13). Therefore, we examined the effect of EGCG on LPS endocytosis in cells. RAW 264 cells were incubated with $10 \mu \mathrm{g} / \mathrm{mL}$ LPS Alexa Fluor 568 in the presence or absence of $100 \mu \mathrm{g} / \mathrm{mL}$ EGCG for $1 \mathrm{~h}$. Then intracellular LPS was measured by a fluorometer. As shown in Fig. 1, EGCG suppressed LPS endocytosis in RAW 264 cells. We next examined the effect of EGCG on LPS-induced innate immune response after its internalization. Nitric oxide (NO) is a mediator of innate immune response, and LPS stimulation increases production of NO in RAW 264 cells (13). Therefore, we examined LPS-induced NO production in cells with DAF-2, a sensitive fluorescent indicator for the detection of NO. As shown Fig. 2, EGCG inhibited production of NO by stimulation with LPS in RAW 264 cell cultures.

\section{EGCG interferes with Rab5-caveolin-1 interaction}

Rab5 is a crucial molecule for the formation of an early endosome (24). Rab5 switches between two distinct conformations, an active state characterized by binding to GTP and an inactive state bound to GDP (20). Rab5Q79L is a constitutively active mutant, because it is defective in GTP hydrolysis and it

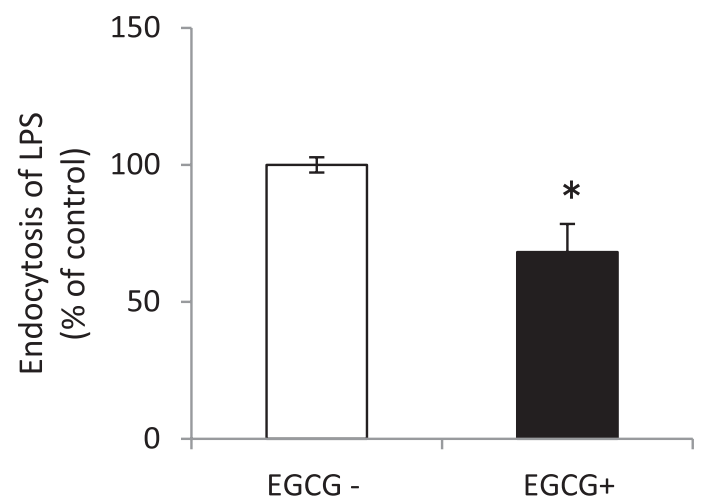

Fig. 1 Effect of EGCG on endocytosis of LPS. RAW 264 cells were incubated with $100 \mu \mathrm{g} / \mathrm{mL}$ EGCG and $10 \mu \mathrm{g} / \mathrm{mL}$ LPS Alexa Fluor 568 for $1 \mathrm{~h}$. Signal intensity of the cell lysate was analyzed with a microplate reader. Each value in the graph is the mean $\pm S D$ of three independent experiments. ${ }^{*} P<0.05$ is locked in an active GTP-bound conformation (15, 22). Rab5S34N is a dominant-negative mutant, because it is defective in GTP binding and is either in a nucleotide-free or guanosine diphosphate (GDP)bound conformation (15). In addition, caveolin-1 interacts with Rab5 and regulates Rab5 activity (10). Therefore, we used HEK293 cells expressing YFPRab5Q79L-CFP-caveolin-1, YFP-Rab5wild-CFP-caveolin-1, and YFP-Rab5S34N-CFP-caveolin-1 and examined the effect of EGCG on the interaction between Rab5 and caveolin-1 in the cells. To investigate the expression of YFP-Rab5 and CFP-caveolin-1 in the three HEK293 cell lines, we first performed Western blotting for these molecules. All of the HEK293 cells stably expressed YFP-Rab5 (Q79L, wild or S34N) and CFP-caveolin-1 (Fig. 3). We also examined the localization of YFP-Rab5 and CFPcaveolin-1 in the HEK293 stable cells by confocal fluorescence microscopy. YFP-Rab5Q79L and YFPRab5 (wild) colocalized with CFP-caveolin-1, while YFP-Rab5S34N did not colocalize with CFP-caveolin-1 (Fig. 4A). Results of FRET analysis showed that the signal intensity (YFP/CFP) in cells expressing YFP-Rab5Q79L and CFP-caveolin-1 was higher than that in cells expressing YFP-Rab5wild and CFP-caveolin-1, whereas the signal intensity in cells expressing YFP-Rab5S34N and CFP-caveolin-1 was lower than that in cells expressing YFP-Rab5wild and CFP-caveolin-1 (Fig. 4B).

Based on results shown in Figs. 3 and 4, we next examined the effect of EGCG on interaction between YFP-Rab5 and CFP-caveolin-1. Results of FRET analysis showed that EGCG reduced the YFP/ CFP emission ratio, indicating a decrease in YFPRab5 and CFP-caveolin-1 interaction (Fig. 5A). Re-

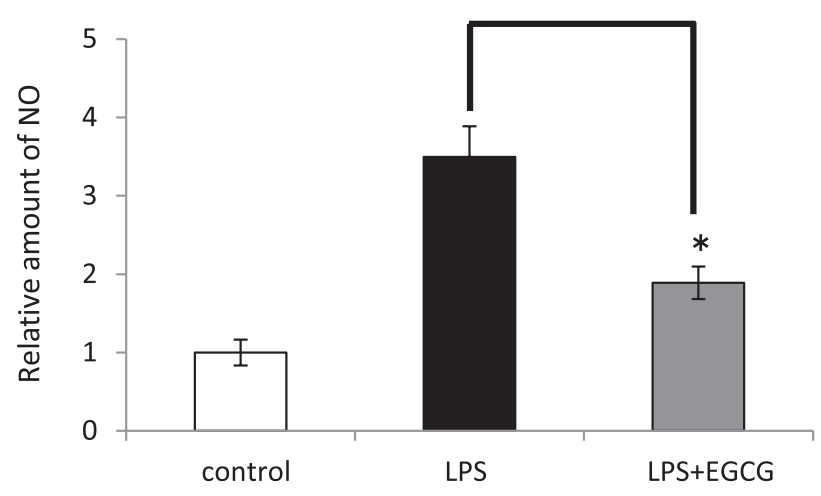

Fig. 2 Effect of EGCG on LPS-induced NO production. RAW 264 cells were incubated with $100 \mu \mathrm{g} / \mathrm{mL}$ EGCG and $100 \mathrm{ng} / \mathrm{mL}$ LPS for $6 \mathrm{~h}$. NO generation was measured with $\mathrm{DAF}-2$. Each value in the graph is the mean $\pm \mathrm{SE}$ of three independent experiments. ${ }^{*} P<0.05$ 
sults of immunoprecipitation using anti-caveolin-1 antibody showed that EGCG also suppressed Rab5caveolin-1 interaction (Fig. 5B). Caveolin-1 binds to the active form of Rab5 and increases Rab5 activity. Therefore, to determine whether EGCG reduces Rab5 activity, we performed a GST-R5BD pull-down assay and measured the level of the active form of Rab5 after treatment with EGCG. As shown in Fig. 5C, Rab5-GTP was decreased by treatment with EGCG. These results suggest that EGCG interferes with the interaction of Rab5 with caveolin-1 by reducing Rab5 activity.

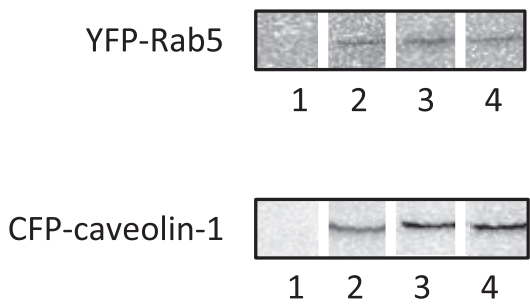

\section{DISCUSSION}

Our results show for the first time that EGCG decreased endocytosis of LPS and LPS-induced production of NO by reducing Rab5-caveolin-1 interaction and Rab5 activity. First, we examined the effect of EGCG on LPS endocytosis in RAW 264 cells. As shown in Fig. 1, EGCG suppressed LPS endocytosis in RAW 264 cells. EGCG suppressed LPS endocytosis dose-dependently (data not shown). We analyzed the interaction between Rab5 and caveolin-1 by using the FRET technique. Results of FRET analysis showed that EGCG reduced the YFP/CFP
1: Negative control
2: YFP-Rab5 S34N + CFP-caveolin-1
3: YFP-Rab5 wild + CFP-caveolin-1
4: YFP-Rab5 Q79L + CFP-caveolin-1

Fig. 3 Expression of YFP-Rab5 and CFP-caveolin-1 in stable transformed HEK 293 cells. Lysates of YFP-Rab5 (S34N, wild, or Q79L) and CFP-caveolin-1 expressing HEK293 cells were analyzed by Western blotting with an anti-Rab5 antibody (upper panel) and an anti-caveolin-1 antibody (lower panel).

(A)
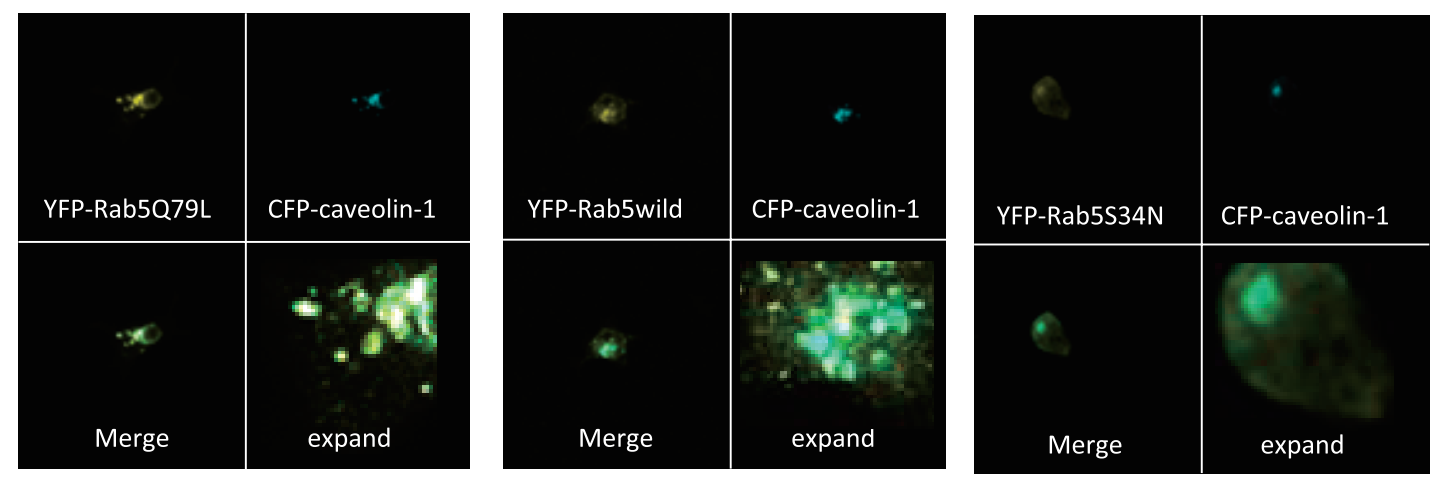

(B)

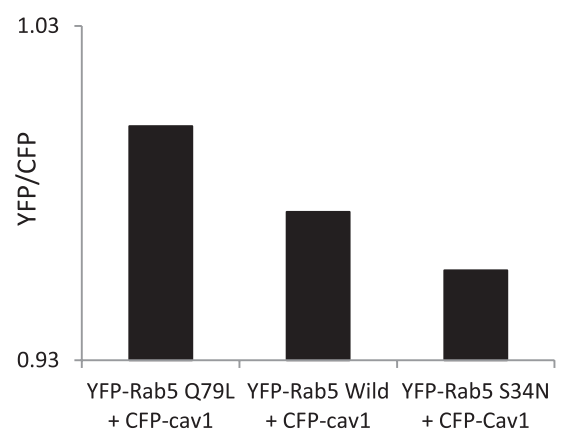

Fig. 4 Localization and signal intensity of YFP-Rab5 and CFP-caveolin-1 in stable transformed HEK 293 cells. (A) YFP-Rab5 and CFP-caveolin-1 in stable transformed HEK 293 cells were observed by using a confocal fluorescence microscope. YFP-Rab5Q79L and YFP-Rab5 wild were colocalized with CFP-caveolin-1, but YFP-Rab5S34N was not. (B) YFP-Rab5 and CFP-caveolin-1-expressing HEK 293 cells were lysed and signal intensity of the cell lysates was measured (YFP/CFP). 
emission ratio, indicating a decrease in YFP-Rab5 and CFP-caveolin-1 interaction. However, we did not clarify whether EGCG directly acts on these molecules or not, because numerous molecules exist in the lysates besides Rab5 and caveolin-1. It has been reported that EGCG directly binds to various plasma proteins including fibronectin, fibrinogen and histidine-rich glycoprotein, Fas, laminin and the 67$\mathrm{kDa}$ laminin receptor, vimentin ZAP-70, Fyn, insulin-like growth factor-I receptor (IGF-IR), and glucose-regulated protein 78 (GRP-78) $(6,7,12,18)$. Thus, EGCG may inhibit Rab5-caveolin-1 interaction via one or more of those proteins. Further stud- ies are needed to determine the manner of EGCG interference with Rab5-caveolin-1 interaction.

EGCG inhibited endocytosis of LPS by simultaneous addition of EGCG and LPS (Fig. 1); however, we have no evidence that EGCG directly binds to LPS. Although we speculate that EGCG inhibits Rab5-caveolin-1 interaction and thereby suppress LPS endocytosis, it is also possible that EGCG directly interferes with LPS binding to the receptor. We will address to this possibility in future studies.

In this study, we investigated the effect of EGCG in cell cultures but not in vivo. A recent study has shown that EGCG suppresses melanoma growth by

(A)

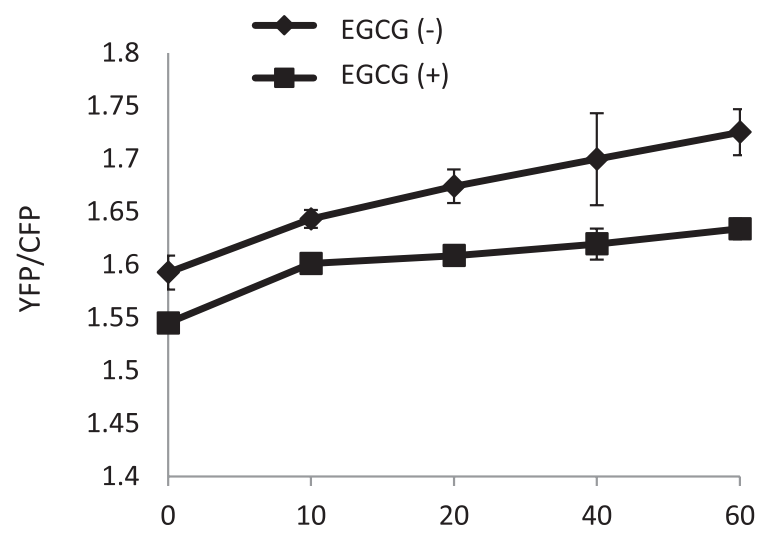

(B)

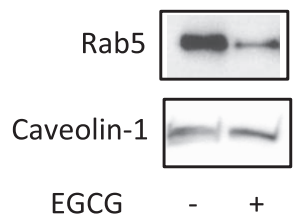

(C)

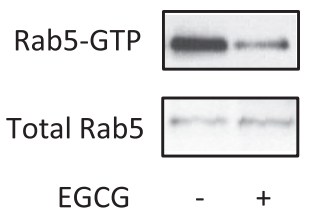

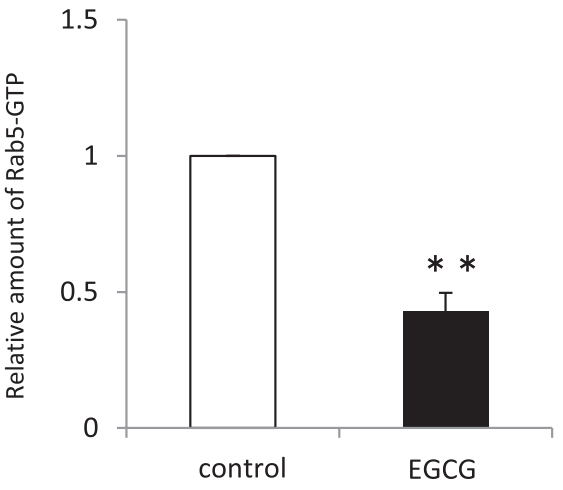

Fig. 5 Effects of EGCG on YFP-Rab5 and CFP-caveolin-1 interaction and Rab5 activity. (A) Lysates of YFP-Rab5 and CFP-caveolin-1-expressing HEK 293 cells were mixed with $100 \mu \mathrm{g} / \mathrm{mL}$ EGCG for $1 \mathrm{~h}$ and then YFP/CFP ratios in the mixtures were measured. (B) HEK 293 cells were treated with $100 \mu \mathrm{g} / \mathrm{mL}$ EGCG for $2 \mathrm{~h}$. Endogenous caveolin-1 in the cell lysates was then immunoprecipitated with an anti-caveolin-1 antibody. Caveolin-1 and Rab5 in the immunoprecipitates were assayed by Western blotting using specific antibodies to caveolin-1 and Rab5. The immunoprecipitated caveolin-1 levels were almost the same with and without EGCG. However, immunoprecipitaed Rab5 level of ECGG (+) was decreased compared with that of ECGG (-). (C) HEK 293 cells were transfected with GFP-Rab5. The cells were treated with $100 \mu \mathrm{g} / \mathrm{mL}$ EGCG for $2 \mathrm{~h}$ and then lysed and subjected to a GST-R5BD pull-down assay. GST-R5BD-bound beads and lysates were assayed by Western blotting with anti-GFP antibody. The total GFP-Rab5 level in the lysate of ECGG (+) was almost the same as that in the lysate of ECGG (-). However, GFP-Rab5-GTP level of ECGG (+) was decreased compared with that of ECGG (-). Rab5-GTP levels were normalized to total GFP-Rab5 levels and quantified using Image J. Each value in the graph is the mean \pm SD of three independent experiments. ${ }^{* *} P<0.01$ 
inhibiting inflammasome and IL-1 $\beta$ secretion in a mouse cancer (5). It has been also shown that EGCG down-regulated the mRNA expression of iNOS, MCP-1 and pro-apoptosis genes and protected testicular seminiferous tubules against ischemia/reperfusion-induced inflammation in the murine testis (1). Furthermore, EGCG has been shown to inhibit EBV-induced B lymphocyte transformation via suppression of RelA acetylation in vivo (4). Therefore, anti-inflammatory effects of EGCG as shown in this study may also be observed in vivo.

It has been reported that LPS increases the production of NO $(3,11,13)$. In this study, EGCG inhibited endocytosis of LPS (Fig. 1) and LPS-induced NO production (Fig. 2). A recent study showed that bacteria-induced production of NO is regulated by endocytosis of bacteria (8). It may be that EGCG inhibits LPS endocytosis and thereby modulates LPS-induced NO generation. Excess NO production followed by iNOS mRNA induction has been shown to augment inflammatory responses and to contribute to the pathogenesis of various diseases, including inflammatory diseases. Therefore, our results raise the possibility that EGCG has beneficial features such as prevention of diseases that involve excessive NO release. In fact, it has been demonstrated that EGCG is beneficial for prevention of and/or therapy for various diseases such as cardiovascular disease, cancer, Parkinson's disease, Alzheimer's disease, cerebral stroke and metabolic diseases (23).

In summary, our results revealed that EGCG reduced LPS-induced innate immune responses through suppression of LPS endocytosis by interfering with Rab5-caveolin-1 interaction and by reducing Rab5 activity. Our results suggest that Rab5 and caveolin-1 are molecular targets of EGCG.

\section{Acknowledgements}

We thank Dr. Y. Yamamoto (Tokyo University of Agriculture) for providing plasmid vectors of YFPRab5Q79L, YFP-Rab5S34N, YFP-Rab5 wild, GFPRab5 wild and CFP-caveolin-1. This work was partly supported by the Sasakawa Scientific Research Grant from The Japan Science Society (to H.M).

\section{REFERENCES}

1. Al-Maghrebi M, Renno WM and Al-Ajmi N (2012) Epigallocatechin-3-gallate inhibits apoptosis and protects testicular seminiferous tubules from ischemia/reperfusion-induced inflammation. Biochem Biophys Res Commun 420, 434-439.

2. Anderson RG (1998) The caveolae membrane system. Annu Rev Biochem 67, 199-225.
3. Carneiro AB, Iaciura BM, Nohara LL, Lopes CD, Veas EM, Mariano VS, Bozza PT, Lopes UG, Atella GC, Almeida IC and Silva-Neto MA (2013) Lysophosphatidylcholine triggers TLR2- and TLR4-mediated signaling pathways but counteracts LPS-induced NO synthesis in peritoneal macrophages by inhibiting NF-kappaB translocation and MAPK/ERK phosphorylation. PLoS One 8, e76233.

4. Choi KC, Jung MG, Lee YH, Yoon JC, Kwon SH, Kang HB, Kim MJ, Cha J H, Kim YJ, Jun WJ, Lee JM and Yoon HG (2009) Epigallocatechin-3-gallate, a histone acetyltransferase inhibitor, inhibits EBV-induced B lymphocyte transformation via suppression of RelA acetylation. Cancer Res 69, 583592.

5. Ellis LZ, Liu W, Luo Y, Okamoto M, Qu D, Dunn JH and Fujita M (2011) Green tea polyphenol epigallocatechin-3-gallate suppresses melanoma growth by inhibiting inflammasome and IL-1beta secretion. Biochem Biophys Res Commun 414, 551-556.

6. Fujimura Y, Sumida M, Sugihara K, Tsukamoto S, Yamada $\mathrm{K}$ and Tachibana H (2012) Green tea polyphenol EGCG sensing motif on the $67-\mathrm{kDa}$ laminin receptor. PLoS One 7, e37942.

7. Gundimeda U, McNeill TH, Elhiani AA, Schiffman JE, Hinton DR and Gopalakrishna R (2012) Green tea polyphenols precondition against cell death induced by oxygen-glucose deprivation via stimulation of laminin receptor, generation of reactive oxygen species, and activation of protein kinase Cepsilon. J Biol Chem 287, 34694-34708.

8. Hagiwara M, Komatsu T, Sugiura SS, Isoda R, Tada H, Tanigawa N, Kato Y, Ishida N, Kobayashi K and Matsushita K (2013) POT1b regulates phagocytosis and NO production by modulating activity of the small GTPase Rab5. Biochem Biophys Res Commun 439, 413-417.

9. Hagiwara M, Shinomiya H, Kashihara M, Kobayashi K, Tadokoro T and Yamamoto Y (2011) Interaction of activated Rab5 with actin-bundling proteins, L- and T-plastin and its relevance to endocytic functions in mammalian cells. Biochem Biophys Res Commun 407, 615-619.

10. Hagiwara M, Shirai Y, Nomura R, Sasaki M, Kobayashi K, Tadokoro T and Yamamoto Y (2009) Caveolin-1 activates Rab5 and enhances endocytosis through direct interaction. Biochem Biophys Res Commun 378, 73-78.

11. Harada K, Shiba T, Doi K, Morita K, Kubo T, Makihara Y, Piattelli A and Akagawa Y (2013) Inorganic polyphosphate suppresses lipopolysaccharide-induced inducible nitric oxide synthase (iNOS) expression in macrophages. PLoS One 8 , e74650.

12. Huang KH, Kuo KL, Chen SC, Weng TI, Chuang YT, Tsai YC, Pu YS, Chiang CK and Liu SH (2012) Down-regulation of glucose-regulated protein (GRP) 78 potentiates cytotoxic effect of celecoxib in human urothelial carcinoma cells. PLoS One 7, e33615.

13. Husebye H, Halaas O, Stenmark H, Tunheim G, Sandanger O, Bogen B, Brech A, Latz E and Espevik T (2006) Endocytic pathways regulate Toll-like receptor 4 signaling and link innate and adaptive immunity. EMBO $J$ 25, 683-692.

14. Kim SJ, Lee JH, Kim BS, So HS, Park R, Myung NY, Um JY and Hong SH (2012) (-)-Epigallocatechin-3-gallate protects against $\mathrm{NO}$-induced ototoxicity through the regulation of caspase-1, caspase-3, and NF-kappaB activation. PLoS One 7, e43967.

15. Li G and Stahl PD (1993) Structure-function relationship of the small GTPase rab5. J Biol Chem 268, 24475-24480.

16. Liu J, Lamb D, Chou MM, Liu YJ and Li G (2007) Nerve 
growth factor-mediated neurite outgrowth via regulation of Rab5. Mol Biol Cell 18, 1375-1384.

17. Olkkonen VM and Stenmark H (1997) Role of Rab GTPases in membrane traffic. Int Rev Cytol 176, 1-85.

18. Patra SK, Rizzi F, Silva A, Rugina DO and Bettuzzi, S (2008) Molecular targets of (-)-epigallocatechin-3-gallate (EGCG): specificity and interaction with membrane lipid rafts. $J$ Physiol Pharmacol 59 Suppl 9, 217-235.

19. Ray A, Cot M, Puzo G, Gilleron M and Nigou J (2013) Bacterial cell wall macroamphiphiles: pathogen-/microbe-associated molecular patterns detected by mammalian innate immune system. Biochimie 95, 33-42.

20. Stan RV (2005) Structure of caveolae. Biochim Biophys Acta
1746, 334-348.

21. Stenmark H (2009) Rab GTPases as coordinators of vesicle traffic. Nat Rev Mol Cell Biol 10, 513-525.

22. Stenmark H, Parton RG, Steele-Mortimer O, Lutcke A, Gruenberg J and Zerial M (1994) Inhibition of rab5 GTPase activity stimulates membrane fusion in endocytosis. EMBO J 13, 1287-1296.

23. Zaveri NT (2006) Green tea and its polyphenolic catechins: medicinal uses in cancer and noncancer applications. Life Sci 78, 2073-2080.

24. Zerial $\mathrm{M}$ and McBride $\mathrm{H}$ (2001) Rab proteins as membrane organizers. Nat Rev Mol Cell Biol 2, 107-117. 